\title{
Involvement of upregulation of fibronectin in the pro-adhesive and pro-survival effects of glucocorticoid on melanoma cells
}

\author{
GAO-XIANG HUANG ${ }^{1,2^{*}}$, MIN-FANG QI ${ }^{1 *}$, XIAO-LONG LI $^{3 *}$, FANG TANG $^{1}$ and LEI ZHU ${ }^{4}$ \\ ${ }^{1}$ Department of Pathology, No. 181 Hospital of People's Liberation Army, Guilin, Guangxi 541002; \\ ${ }^{2}$ Department of Pathophysiology, Second Military Medical University, Shanghai 200433; ${ }^{3}$ Department of Cardiology, \\ Navy General Hospital, Beijing 100048; ${ }^{4}$ Department of Orthopedic Trauma Surgery, Changzheng Hospital, \\ Second Military Medical University, Shanghai 200003, P.R. China
}

Received August 26, 2017; Accepted December 5, 2017

DOI: $10.3892 / \mathrm{mmr} .2017 .8269$

\begin{abstract}
Glucocorticoids (GCs) are important stress hormones, which are used as a concomitant medication during malignant tumor chemotherapy. Clinical and preclinical studies have linked GCs to melanoma growth and progression. However, the effects and mechanism of action of GCs on the adhesion and survival of melanoma cells are still unknown. In the present study the effect of dexamethasone (Dex), a synthetic GC, on fibronectin (FN) expression and its roles in regulating the adhesion and survival of melanoma cells were investigated. It was revealed that Dex significantly increased the levels of intracellular and secreted FN in melanoma cell lines by increasing glucocorticoid receptor-mediated FN protein stability. Additionally, it was demonstrated that Dex (100 $\mathrm{nM})$ significantly promoted the adhesion and survival of melanoma cells. Silencing FN expression abrogated the pro-adhesive and pro-survival effects of Dex in melanoma cells. Extracellular FN significantly enhanced melanoma cell adhesion and survival in the presence of cisplatin, whereas partially blocking extracellular FN signaling with a CD44 antibody significantly reduced $\mathrm{FN}$-enhanced adhesion and survival. This indicated that the upregulation of FN contributed to the pro-survival effect of Dex by enhancing cell adhesion. It was also observed that activation of the PI3K/AKT signaling pathway by extracellular FN was involved in the FN-mediated
\end{abstract}

Correspondence to: Dr Lei Zhu, Department of Orthopedic Trauma Surgery, Changzheng Hospital, Second Military Medical University, 415 Fengyang Road, Shanghai 200003, P.R. China

E-mail: hailangzhulei@smmu.edu.cn

*Contributed equally

Abbreviations: GC, glucocorticoid; GR, glucocorticoid receptor; Dex, dexamethasone; FN, fibronectin; CHX, cycloheximide; ECM, extracellular matrix

Key words: dexamethasone, fibronectin, melanoma, cell adhesion, cell survival increase in melanoma cell survival. These findings increase understanding of the possible mechanisms by which GCs regulate melanoma cell adhesion and survival.

\section{Introduction}

Clinical and preclinical studies have shown that chronic stress has an impact on tumor growth and progression (1-4). As important stress hormones, glucocorticoids (GCs) influence tumor biology not only through their systematical immunosuppressive and anti-inflammatory effects $(5,6)$, but also by changing the tumor microenvironment and playing a direct role in regulating the proliferation, metabolism, differentiation and apoptosis of tumor cells (7). Moreover, synthetic GCs, such as dexamethasone (Dex), have been widely used as concomitant medications to reduce acute toxicity and alleviate side effects, such as hyperemesis induced by chemotherapy or radiotherapy in non-hematologic cancer therapy $(7,8)$. GCs are also given to patient before, during and after chemotherapy of solid malignant tumors to protect normal tissue, e.g., bone marrow progenitor cells, against the long-term effects of genotoxic drugs (9). Recently, emerging evidence has shown that GCs exert inhibitory effects on tumor apoptosis induced by chemotherapeutics not only in established cancer cell lines and tumor xenografts, but also in freshly isolated cells from surgical specimens, such as breast, ovary, prostate, liver and skin (10-13). Therefore, it is important to consider the clinical relevance of the survival-promoting effects of GCs when they interfere with chemotherapeutics.

The effects of GCs are mediated by the glucocorticoid receptor (GR), which is ubiquitously expressed in all cell, and exerts its biological effects by regulating the expression of genes and cross-talking with multiple trans-membrane signaling pathways (14). An increasing number of studies have reported that GCs/GR promote cancer cell survival in an unfavorable microenvironment and enhance the resistance of solid tumors to chemotherapy by regulating the expression of genes and activating trans-membrane signaling pathways, which is very pivotal for the cancer progression (15). The pro-survival and anti-apoptotic effects of GCs are meditated by GR through regulation of the expression of genes, such as inhibitors of apoptosis (cIAP-2, X-IAP, Bcl-XL, and Bcl-2), mitogen-activated 
protein kinase phosphatase-1 (MKP-1), as well as serum- and glucocorticoid-inducible kinase 1 (SGK-1) $(16,17)$.

Cell adhesion to the extracellular matrix (ECM) is pivotal for survival and growth of most of solid cancer cells and is mediated by several cell surface adhesion molecules such as integrin $\beta 1$ and CD44 and their ligands, which are ECM components, such as collagens, fibronectin (FN) and laminin $(18,19)$. Binding of the key ECM protein FN to cell surface adhesion molecules not only supports cell adhesion, but also brings cytoplasmic molecules together to form protein-rich focal complexes that activate focal adhesion kinase (FAK) and several intracellular signaling molecules and pathways, such as Rho GTPases, Ras GTPase, Src and the PI3K-Akt pathway, that regulate cell proliferation, survival, spreading and migration (18-22). So far, studies on the regulation of FN by GCs and its role in GC-induced pathophysiological process are limited. Ahadome et al reported that Dex increases FN expression in ocular trabecular meshwork cells, and this increase in FN expression is involved in the steroid-induced glaucoma (23), while another study reported Dex negatively regulates FN expression in cytotrophoblasts isolated from human placenta (24). However, due to limited data it is unclear whether the regulation of FN expression by GCs is cell type-dependent.

Melanoma is characterized by frequent recurrence and high mortality in skin cancers. Human melanoma cells express high-affinity GR (25). Previous studies have shown that GCs have no significant direct effects on the proliferation of most human melanoma cells in vitro, but giving liposomal prednisolone phosphate (PLP) for prolonged periods of time reduces the melanoma growth by inhibiting tumor angiogenesis in mice $(25,26)$. Recently a clinicopathological study demonstrated that the subcellular distribution of GR in cutaneous melanoma specimens is associated with tumor thickness and Clark level, the level of anatomical invasion of melanoma in the skin (27). However, it is still unclear whether GCs affect the adhesion and survival of melanoma cells.

In this study, we found that Dex, a synthetic GC, significantly upregulated FN expression and increased its secretion in melanoma cells. We further investigated the mechanism and biological significance of FN upregulation by Dex in melanoma cells. This study facilitates understanding the mechanism by which GCs affect melanoma biology, especially the adhesion and survival of melanoma cells.

\section{Materials and methods}

Cell culture. Human A375 melanoma cells and murine B16F10 melanoma cells were obtained from the Cell Bank of the Chinese Academy of Sciences (Shanghai, China). The two cell lines were routinely cultured in RPMI-1640 (Gibco, USA) containing $10 \%$ fetal bovine serum (FBS, Bioind, Israel) and maintained in an incubator with a humidified atmosphere of $5 \% \mathrm{CO}_{2}$ at $37^{\circ} \mathrm{C}$. Wortmannin, cisplatin, cycloheximide $(\mathrm{CHX})$ and RU486 were purchased from Sigma-Aldrich (Merck KGaA, Darmstadt, Germany) and dissolved in dimethyl sulfoxide (DMSO) or ethanol. Recombinant human FN was purchased from R\&D Systems, Inc. (Minneapolis, MN, USA). For Dex treatment, cells were cultured in medium containing $10 \%$ Dextran-coated charcoal (DCC)-treated FBS to avoid possible interference from serum steroids and incubated with 100 nM Dex (Sigma-Aldrich; Merck KGaA) for different periods of time. Control cells were incubated with ethanol vehicle $(<1 \%$ v/v).

$R N A$ extraction and reverse transcription-quantitative polymerase chain reaction ( $R T-q P C R)$. Total RNA was isolated using TRIzol reagent (Invitrogen; Thermo Fisher Scientific, Inc., Waltham, MA, USA), and $2 \mu \mathrm{g}$ total RNA was reverse transcribed using Reverse Transcription Reagents (Takara Bio, Inc., Otsu, Japan) following the manufacturer's protocol. RT-qPCR was performed in triplicate using SYBR Green PCR Master Mix (Toyobo Life Science, Osaka, Japan) on a Mastercycler ep realplex (Eppendorf, Hamburg, Germany). The primer sequences used were as follows. FN (human): 5'-CGGTGGCTGTCAGTCAAAG-3' (forward) and 5'-AAA CCTCGGCTTCCTCCATAA-3' (reverse). FN (mouse): 5'-GTCAGTGTCTCCAGTGTCTAC-3' (forward) and 5'-TGG CTTGCTGGCCAATCAGT-3' (reverse). GAPDH (human): 5'-CATGAGAAGTATGACAACAGCCT-3' (forward) and 5'-AGTCCTTCCACGATACCAAAGT-3' (reverse). $\beta$-actin (mouse): 5'-CTGTATGCCTCTGGTCGTAC-3' (forward) and 5'-TGATGTCACGCACGATTTCC-3' (reverse). Thermal cycling conditions consisted of an initial denaturing step $\left(95^{\circ} \mathrm{C}, 2 \mathrm{~min}\right)$ followed by 40 cycles of denaturing $\left(95^{\circ} \mathrm{C}\right.$, $15 \mathrm{sec})$, annealing $\left(60^{\circ} \mathrm{C}, 15 \mathrm{sec}\right)$ and extending $\left(72^{\circ} \mathrm{C}\right.$, $45 \mathrm{sec})$. The level of FN mRNA was normalized to GAPDH or $\beta$-actin (internal control), and relative quantification was done using the $2^{\Delta \Delta \mathrm{Cq}}$ formula. Changes in gene expression were expressed as the relative fold-increase in mRNA compared with a control.

Western blotting. Total cell lysates were prepared with 1x SDS lysis buffer with $100 \mathrm{mM}$ Dithiothreitol and $2 \mu \mathrm{g} / \mathrm{ml}$ protease inhibitor solution containing $0.1 \mathrm{mM}$ each leupeptin, aprotinin, and pepstatin. After electrophoresis, proteins were transferred to nitrocellulose membrane, blocked with $5 \%$ (v/v) nonfat milk in tris-buffered saline Tween-20 (TBST), and probed overnight at $4^{\circ} \mathrm{C}$ with primary antibodies against FN (sc-6953, 1:500; Santa Cruz Biotechnology, Inc., Dallas, TX, USA), $\beta$-actin (1:10,000, Sigma-Aldrich; Merck KGaA), or p-Akt1/2/3 (Ser 473) (sc-7985, 1:1,000; Santa Cruz Biotechnology, Inc.), Akt1/2/3 (sc-8312, 1:1,000; Santa Cruz Biotechnology, Inc.). Then the membranes were washed three times and incubated with HRP-conjugated secondary antibodies (1:5,000; Rockland Immunochemicals, Inc., Gilbertsville, PA, USA) for $2 \mathrm{~h}$. Finally HRP was detected using enhanced chemiluminescence (Pierce; Thermo Fisher Scientific, Inc.). Protein bands were quantified with ImageJ software (National Institutes of Health, Bethesda, MD, USA) using $\beta$-actin as an internal control.

ELISA. A375 melanoma cells were treated with or without $100 \mathrm{nM}$ Dex for the indicated times, and then the conditioned media were collected and analyzed using human FN ELISA kits (R\&D Systems, Inc.) according to the manufacturer's instructions. Absorbance of samples was read at $450 \mathrm{~nm}$ in a UV-visible spectrophotometer. The protein concentration was calibrated from a dose response curve based on reference standards. 
RNA interference. SiRNAs were manufactured by GenePharma Co., Ltd. (Shanghai, China). The sequences were as follows. FN siRNA: 5'-GCAGUGGCUGAAGACACAAGGAAAU-3'; Control siRNA: 5'-CGCTTACCGATTCAGAATGG-3'. A375 cells were transfected with a final concentration of $10 \mathrm{nmol} / \mathrm{l}$ FN siRNA or control siRNA using INTERFERin ${ }^{\mathrm{TM}}$ (Polyplus Transfection, Strasbourg, France) following the manufacturer's instructions.

Cell adhesion assay. Cell adhesive ability was determined by cell adhesion assay. After cells were treated with Dex or transiently transfected with siRNA for the indicated times, cells were digested into single cell suspension and $8 \times 10^{4}$ cells were seeded onto non-coated 96 -well plates in triplicate and incubated at $37^{\circ} \mathrm{C}$ for $60 \mathrm{~min}$. The plates were gently washed thrice with 1X PBS to remove the non-adherent cells. The number of remaining cells in the 96 -well plates was determined by 3-(4,5-dimethylthiazol-2-yl)-2,5-diphenyltetrazolium bromide (MTT) assay. Briefly, the remaining cells were incubated in medium supplemented with $50 \mu \mathrm{g} / \mathrm{ml}$ methylthiazole tetrazolium for $3 \mathrm{~h}$. Cells were then solubilized by adding $200 \mu 1$ DMSO. The absorbance was measured at $570 \mathrm{~nm}$ in a UV-visible spectrophotometer.

Cell adhesion ability was also determined in 96-well plates coated with or without human FN purchased from CORNING (USA). Before the cell adhesion assay, cells were pre-treated with a CD44 primary antibody (103014; BioLegend, Inc., San Diego, CA, USA) and Con IgG antibody (400622; BioLegend, Inc.) for $1 \mathrm{~h}$.

Analysis of cell viability. Cells were seeded in 96-well culture plates at a density of $3 \times 10^{3}$ cells per well in triplicate, allowed to attach overnight, and then treated with the indicated chemicals or reagents. Cisplatin, a chemotherapeutic drug applied in melanoma therapy, was used to induce cell death. At the indicated time, cell viability was evaluated using the Cell Counting Kit-8 (CCK-8; Dojindo Molecular Technologies, Inc., Kumamoto, Japan) following the standard procedures provided by the manufacturer. The optical density (OD) was measured at a wavelength of $450 \mathrm{~nm}$ using a Labsystem multiskan microplate reader (Merck Eurolab, Dietikon, Switzerland).

Statistical analysis. Statistical significances between multiple experimental groups were analyzed by one-way analysis of variance and Tukey's post hoc tests. The Student's t-test was used to compare the difference between two different groups. $\mathrm{P}<0.05$ was considered to indicate a statistically significant difference. Quantitative data were expressed as the mean \pm standard deviation of at least three determinations.

\section{Results}

Dex increased the expression and secretion of FN in melanoma cells. We first examined the expression of FN in A375 and B16F10 melanoma cells treated with $100 \mathrm{nM}$ Dex for different periods of times by RT-qPCR and western blotting. We did not observe a significant change in the level of FN mRNA (Fig. 1A and B), but we found that Dex significantly upregulated FN protein in A375 and B16F10 cells in a time-dependent fashion, with the maximal induction of FN protein (3.9-fold of control and 3.5-fold of control, respectively, $\mathrm{P}<0.01$ ) observed at $48 \mathrm{~h}$ after Dex treatment (Fig. 1C and D). A significant increase in the level of secreted FN was also observed at $48 \mathrm{~h}$ and $72 \mathrm{~h}$ after Dex treatment in A375 cells (Fig. 1E).

Upregulation of FN expression by Dex was due to a $G R$-mediated increase in protein stability. Since upregulation of FN by Dex occurred at the post-transcriptional level, we investigated whether Dex-induced upregulation of FN was mediated through GR and caused by an increase in the protein stability in A375 melanoma cells. As shown in Fig. 2A, RU486, an antagonist of GR, dramatically reversed the upregulation of FN protein, indicating that the effect of Dex was mediated through GR.

We next examined the protein stability of FN in the presence and absence of Dex by western blotting. A375 cells were pre-treated with Dex or vehicle for $48 \mathrm{~h}$ and further treated with $100 \mu \mathrm{g} / \mathrm{ml}$ cycloheximide (CHX, a protein synthesis inhibitor) for different amounts of times. As shown in Fig. 2B, Dex significantly extended the half-life of FN protein (from 21.11 to $39.87 \mathrm{~h}$, a 1.89 -fold increase, $\mathrm{P}<0.01$ ). These results indicate that Dex-induced upregulation of FN was achieved by preventing protein degradation.

Upregulation of FN contributed to the pro-adhesive effect of Dex in melanoma cells. As a multifunctional ECM glycoprotein and a core component of many extracellular matrices, FN plays an important role in regulating intracellular signal transduction and tumor behaviors, including cell adhesion and cell survival (28-30). Therefore, we investigated whether the effect of Dex on melanoma biology was due to upregulation of FN. We silenced the expression of FN using specific small RNA interference (si-FN) and determined the effects of knock-down of FN on melanoma cell adhesion. We found that $100 \mathrm{nM}$ Dex significantly enhanced the adhesive ability of B16F10 and A375 cells (Fig. 3A). Western blotting confirmed that knock-down of FN expression with si-FN almost abolished the Dex-induced expression of FN in A375 cells (Fig. 3B). As shown in Fig. 3C, Dex significantly enhanced the adhesive ability of A375 cells transfected with control small RNA interference (si-con). However, the pro-adhesive effect of Dex in FN knock-down cells was almost completely inhibited, indicating that upregulation of FN contributed to the pro-adhesive effect of Dex in melanoma cells.

In order to further evaluate the role of $\mathrm{FN}$ in the adhesion of A375 cells, we examined cell adhesive capacity on uncoated and FN-coated wells (to imitate the over-expression of extracellular FN). The number of adhering cells in the FN-coated group was significantly higher than the uncoated control group (Fig. 3D), indicating that augmentation of extracellular FN enhanced A375 cell adhesive capacity.

Upregulation of FN contributed to the pro-survival effect of Dex by enhancing melanoma cell adhesion. Studies have shown that cell adhesion to the ECM is pivotal for survival and growth of most of solid malignant cells, so we further investigated the role of FN upregulation in the effect of Dex on melanoma cell survival in the presence of the chemotherapeutic agent cisplatin. We found that treatment with cisplatin 


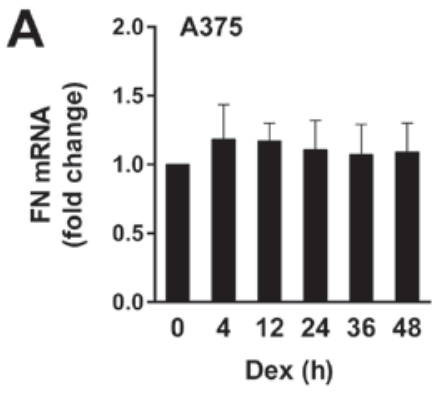

C A375

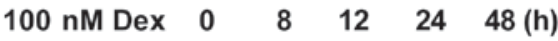
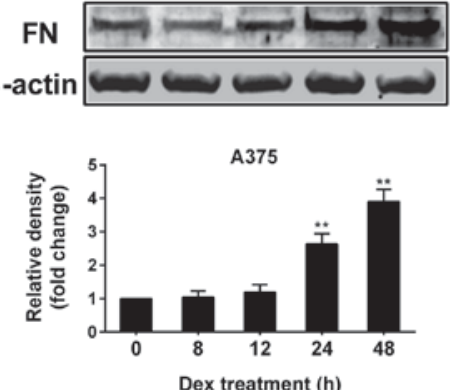

B

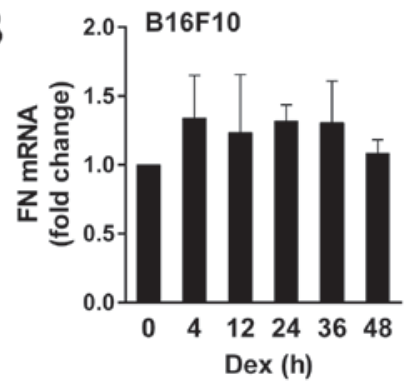

D B16F10

$\begin{array}{llllllll}100 & \text { nM Dex } & 0 & 12 & 24 & 36 & 48 & \text { (h) }\end{array}$
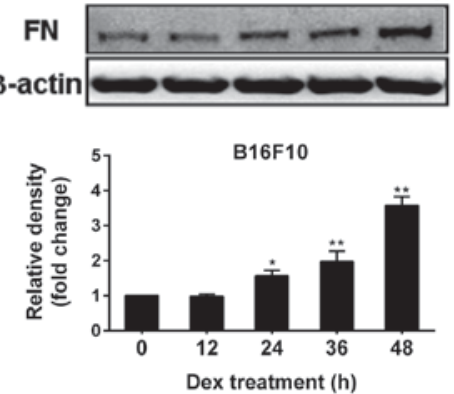

E

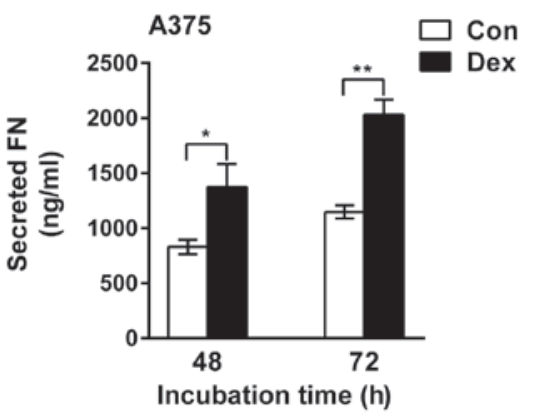

Figure 1. Effect of Dex on the expression and secretion of FN in melanoma cells. A375 and B16F10 melanoma cells were treated with Dex (100 nM) for the indicated times, and then the levels of (A and B) FN mRNA and (C and D) protein were assessed by RT-qPCR and western blotting, respectively. The protein bands were quantified by densitometric analysis. (E) The levels of secreted FN in A375 cells incubated with or without Dex (100 nM) for the indicated times were determined by ELISA as described in the Materials and methods. The concentration of FN in cell culture supernatants was calibrated from a dose response curve based on reference standards. The values are expressed as the fold change relative to the vehicle-treated control and represent the mean of three separate experiments. Each bar represents the mean \pm standard deviation. ${ }^{*} \mathrm{P}<0.05,{ }^{* *} \mathrm{P}<0.01$ vs. the vehicle-treated control.

A

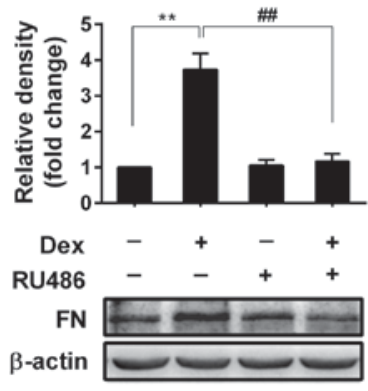

B
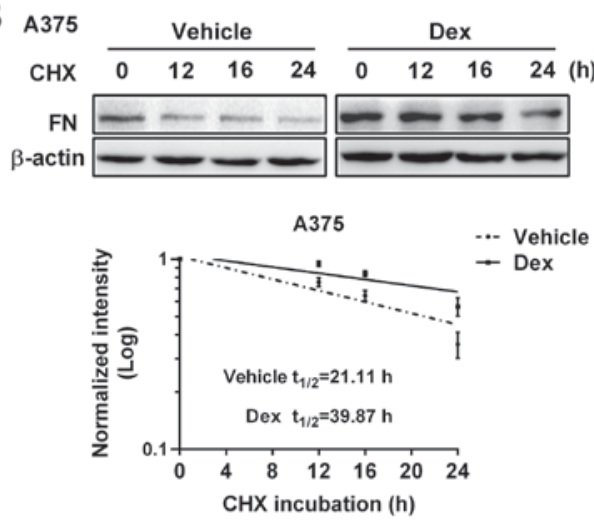

Figure 2. Impact of Dex on the protein stability of FN in melanoma cells. (A) A375 cells were pre-incubated with or without GR antagonist RU486 (50 $\mu$ M) for $1 \mathrm{~h}$ prior to Dex $(100 \mathrm{nM})$ treatment for $48 \mathrm{~h}$. The accumulation of FN protein was assessed by western blotting and $\beta$-actin was used as a loading control. The protein bands were quantified by densitometric analysis. (B) Following Dex $(100 \mathrm{nM})$ or vehicle treatment for $48 \mathrm{~h}, \mathrm{~A} 375$ cells were treated with cycloheximide (CHX, $100 \mu \mathrm{g} / \mathrm{ml})$ and maintained in medium containing Dex (100 $\mathrm{nM}$ ) or vehicle for 12, 16 and $24 \mathrm{~h}$, respectively. The level of FN protein was assessed by western blotting. FN protein bands were quantified by densitometric analysis using $\beta$-actin as an internal control. A Log-normal plot of normalized FN level is shown (below) and the half-life of FN protein was evaluated by fitting a first-order exponential curve to the data points $\left(\mathrm{R}^{2}=0.8988\right.$ in the vehicle group, $\mathrm{R}^{2}=0.7195$ in the Dex group). The values at each time point represent the means \pm standard deviation of one independent experiment performed in triplicate. ${ }^{* * *} \mathrm{P}<0.01$ vs. the vehicle-treated control, ${ }^{\# \#} \mathrm{P}<0.01$ vs. Dex-treated cells. 

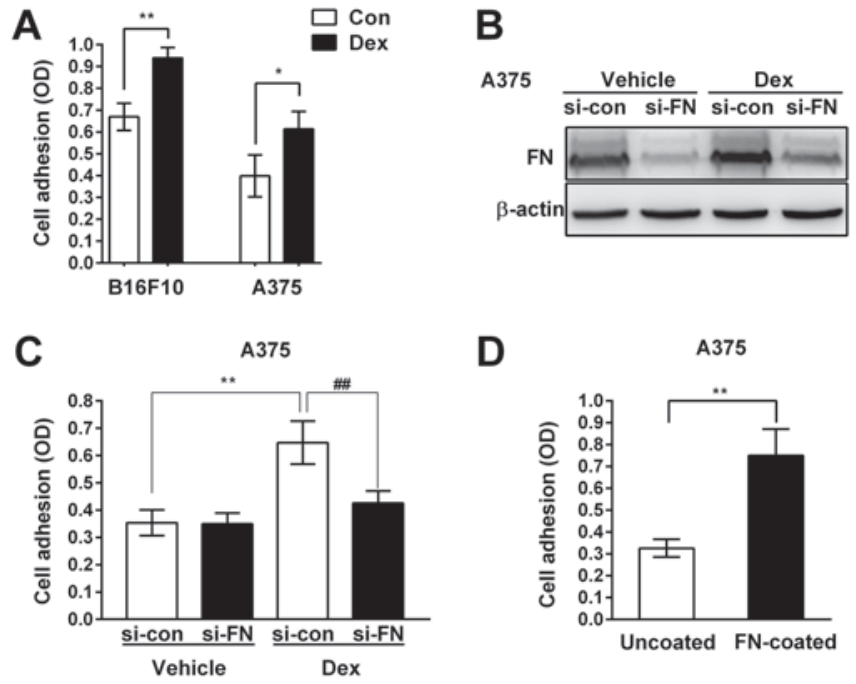

Figure 3. Upregulation of FN mediated the effect of Dex on the adhesion of melanoma cells. (A) B16F10 and A375 melanoma cells were pre-incubated with Dex $(100 \mathrm{nM})$ or vehicle for $48 \mathrm{~h}$, and then cell adhesion was assayed with uncoated 96-well plates as described in the Materials and Methods. (B) A375 cells were transfected with FN siRNA (si-FN) or Con siRNA (si-con) for $24 \mathrm{~h}$, and then transfected cells were continuously treated with or without Dex $(100 \mathrm{nM})$ for another $48 \mathrm{~h}$. Knock-down of FN expression was monitored at the protein level by western blotting, and $\beta$-actin was used as a loading control. (C) A transfected single cell suspension was prepared and cell adhesion was assayed with uncoated 96-well plates. (D) $8 \times 10^{4}$ cells were seeded onto 96-well plates coated with or without human FN $(10 \mu \mathrm{g} / \mathrm{ml})$, and then cell adhesion was assayed. Data are summarized from three independent experiments. Each bar represents the mean \pm standard deviation. ${ }^{*} \mathrm{P}<0.05$, ${ }^{* *} \mathrm{P}<0.01$ vs. vehicle-treated cells $(\mathrm{A})$, vehicle-treated Con siRNA cells (C) or the uncoated control (D), ${ }^{\# \#} \mathrm{P}<0.01$ vs. Dex-treated Con siRNA cells (C).

for $24 \mathrm{~h}$ decreased A375 cell viability in a dose-dependent manner (Fig. 4A). The survival rate of A375 cells treated with $40 \mu \mathrm{M}$ cisplatin was decreased to $39 \%$ ( $\mathrm{P}<0.01)$ (Fig. 4B). However, we found that $100 \mathrm{nM}$ Dex significantly weakened the cytotoxic effect of cisplatin and increased the cell survival rate to $68 \%(\mathrm{P}<0.01)$ (Fig. 4B). Similar results were observed for B16F10 cells (Fig. 4C), indicating that Dex exerted a pro-survival effect on melanoma cells under unfavorable conditions (Fig. 4B and C). Next, we observed the effect of Dex and cisplatin treatment on survival in cells where FN expression was silenced by si-FN. We found that Dex also enhanced cell resistance to cisplatin in A375 cells transfected with si-con (Fig. 4D), but inhibiting FN expression with si-FN significantly reduced the pro-survival effect of Dex in the presence of cisplatin (Fig. 4D). These results indicate that upregulation of FN contributes to Dex-induced melanoma cell survival and chemo-resistance.

The interaction of extracellular FN with one of its receptors, such as CD44, mediates cell adhesion by triggering several signaling pathways, and adhesion consequently regulates cell behaviors, including cell survival $(31,32)$. Therefore, we further investigated the correlation between FN-enhanced cell adhesion and FN-enhanced melanoma cell survival and resistance to chemotherapeutics. We used FN-coated wells to mimic extracellular FN. As shown in Fig. 4E, blocking CD44 signaling with an anti-CD44 antibody significantly abrogated the effect of FN-enhanced cell adhesion in A375 cells, suggesting the partial involvement of extracellular FN/CD44 signaling in enhanced cell adhesion. Furthermore, extracellular
FN protected A375 cells against chemotherapeutics, further validating the effect of FN on enhanced cell survival under unfavorable conditions (Fig. 4F). Inhibiting extracellular FN signaling with an anti-CD44 antibody not only reduced cell adhesion, but also significantly reduced the FN-mediated pro-survival effect in the presence of cisplatin (Fig. 4F). These data indicate that cell adhesion is positively linked to melanoma cell survival and that Dex-induced survival and chemo-resistance is mediated through FN upregulation and enhancement of cell adhesion.

PI3K/AKT activation contributed to FN-mediated melanoma cell survival. The PI3K/AKT pathway plays a critical role in modulating tumor cell proliferation, adhesion and survival (33-35). Therefore, we examined whether the PI3K/AKT pathway was involved in the pro-survival effect of FN on melanoma cells. We found that treatment with recombinant human FN (rh-FN; $10 \mu \mathrm{g} / \mathrm{ml}$ ) significantly increased the level of phosphorylated AKT at S473 in A375 cells in a time-dependent manner (Fig. 5A). Treatment with rh-FN also significantly increased cell viability from 36 to $61 \%(\mathrm{P}<0.01)$ (Fig. 5B). However, inhibiting the PI3K/AKT pathway with wortmannin almost abolished the pro-survival effect of rh-FN in the presence of cisplatin in A375 cells (Fig. 5B). These results indicate that activation of PI3K/AKT by extracellular $\mathrm{FN}$ is involved in the survival and enhancement of cisplatin resistance in melanoma cells.

\section{Discussion}

GCs are important stress hormones and are used as a concomitant medication during malignant tumor chemotherapy. Current studies have suggested a positive relationship between GCs and melanoma progression (27,36-38). The mechanism by which GC influences melanoma biology is still unclear. In this study, we investigated the role of Dex in regulating FN expression, and the biological significance of this regulation in melanoma cells. We found that Dex, a synthetic GC, significantly increased the level of FN protein in melanoma cells, including its secreted form. Upregulation of FN protein by Dex was mediated through GR, which acted post-transcriptionally by increasing FN protein stability. GC was previously reported to increase FN synthesis and induce FN matrix assembly in normal human fibroblasts, HT-1080 fibrosarcoma cells and chick hepatocytes, but GC was found to negatively regulate FN expression in placenta $(24,39-41)$. These contrasting results suggest that the regulation of $\mathrm{FN}$ expression by $\mathrm{GC}$ is dependent on cell type.

$\mathrm{FN}$ is a multifunctional ECM glycoprotein and a core component of many extracellular matrices, and plays an essential role in regulating epithelial cell adhesion to the ECM (42). In the present study, we found that Dex significantly increased the levels of both intracellular and secreted $\mathrm{FN}$ protein in melanoma cells and promoted adhesion. Knock-down of FN expression in melanoma cells significantly reduced the pro-adhesive effect of Dex. Furthermore, we demonstrated that the addition of extracellular FN enhanced the adhesive capacity of melanoma cells. These results indicate that upregulation of FN mediates the pro-adhesive effect of Dex on melanoma cells. 

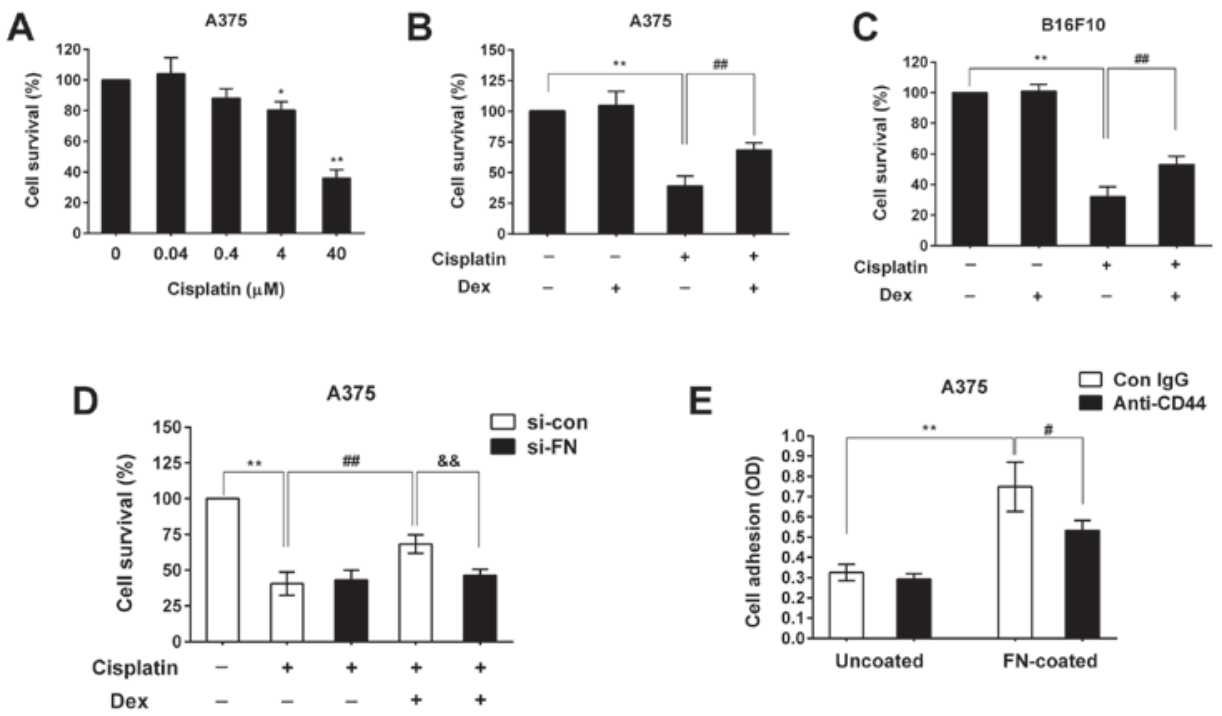

E
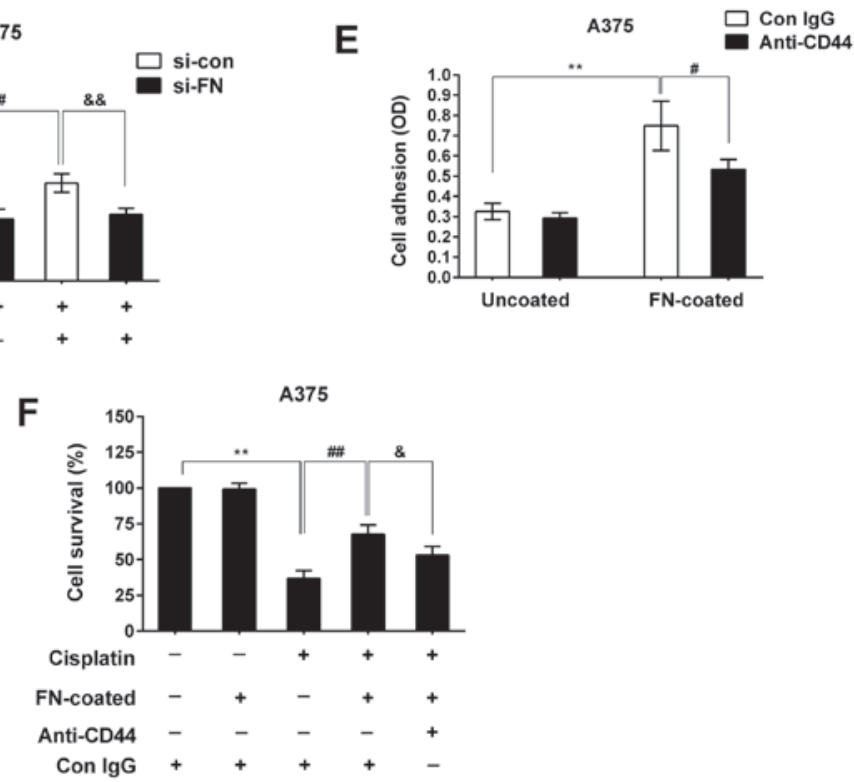

Figure 4. Upregulation of FN mediated the pro-survival effect of Dex by enhancing the adhesion of melanoma cells. (A) A375 cells were treated with different dose of cisplatin for $24 \mathrm{~h}$, and then cell viability was analyzed using the CCK-8 kit. (B) A375 cells, (C) B16F10 cells and (D) transfected A375 cells were pre-incubated with or without Dex $(100 \mathrm{nM})$ for $24 \mathrm{~h}$ and then cultured continuously in the presence or absence of cisplatin $(40 \mu \mathrm{M})$ for another $24 \mathrm{~h}$. Cell viability was analyzed as described in the Materials and Methods. (E) Following pre-incubation with a CD44-blocking antibody (anti-CD44, $40 \mu \mathrm{g} / \mathrm{ml}$ ) or Con $\mathrm{IgG}$ antibody for $1 \mathrm{~h}, \mathrm{~A} 375$ cells were seeded onto 96-well plates coated with or without human FN $(10 \mu \mathrm{g} / \mathrm{ml})$. Cell adhesion was assayed as described in the Materials and methods. (F) Cells were further treated with cisplatin $(40 \mu \mathrm{M})$ for $24 \mathrm{~h}$, and cell viability was measured. The values represent means \pm standard deviation of three separate experiments. ${ }^{*} \mathrm{P}<0.05,{ }^{* *} \mathrm{P}<0.01$ vs. the vehicle-treated control, ${ }^{\# \#} \mathrm{P}<0.01$ vs. cisplatin-treated cells, ${ }^{\#} \mathrm{P}<0.05$ vs. the FN-coated control (E), ${ }^{\&} \mathrm{P}<0.05,{ }^{\& \&} \mathrm{P}<0.01$ vs. cisplatin-treated cells with Dex or cisplatin-treated cells with FN.

A

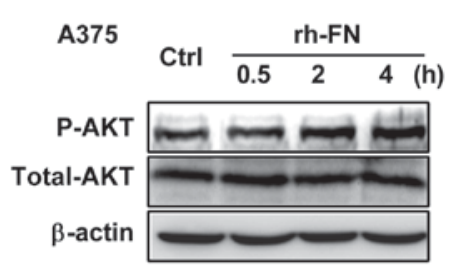

B

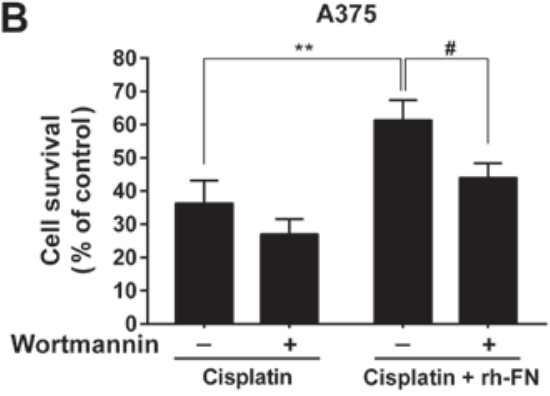

Figure 5. Activation of PI3K/AKT was involved in the FN-induced survival of melanoma cells. (A) A375 cells were treated with recombinant human FN (rh-FN, $10 \mu \mathrm{g} / \mathrm{ml}$ ) for the indicated times, and then the protein levels of p-AKT (S473) and total AKT were detected by western blotting. (B) A375 cells were pre-treated with rh-FN $(10 \mu \mathrm{g} / \mathrm{ml})$ and/or wortmannin $(100 \mathrm{nM})$ for $1 \mathrm{~h}$ prior to cisplatin $(40 \mu \mathrm{M})$ treatment for another $24 \mathrm{~h}$. Cell viability was analyzed using the CCK-8 kit. The values represent means \pm standard deviation of three separate experiments. ${ }^{* *} \mathrm{P}<0.01$ vs. cisplatin-treated cells, ${ }^{*} \mathrm{P}<0.05$ vs. cisplatin-treated cells with rh-FN.

Cell adhesion to the ECM is pivotal for survival and growth of most solid malignant cells $(18,19)$. Consistent with this, we demonstrated that in addition to regulating the adhesion of melanoma cells, Dex increased the survival and chemo-resistance of melanoma cells during cisplatin-induced cell death. Knock-down of FN expression not only reduced cell adhesive capacity, but also abrogated the pro-survival effect of Dex; therefore, we hypothesize that upregulation of FN by Dex enhances cell adhesion, thereby enhancing cell survival under unfavorable conditions. CD44, a broadly distributed transmembrane glycoprotein, mediates cell-matrix interactions through binding to some ECM components, such as FN, collagens and laminin. To test our hypothesis, an anti-CD44 antibody was used to block the extracellular FN signaling and 
inhibit FN-enhanced cell adhesion. We found that anti-CD44 antibody treatment only partially blocked the FN-mediated increase in melanoma cell survival in the presence of cisplatin, indicating that $\mathrm{FN}$ binding to other receptors in addition to CD44 may contribute to the pro-survival effect of Dex.

It is known that increased adhesion mediated by FN-receptor interaction plays an essential role in regulating cell proliferation, survival and migration by triggering several signaling pathways, especially the PI3K/AKT pathway, which is the most important pathway promoting cell survival (43). Interaction of CD44 with its ligands, including FN, can also enhance proliferation, survival and invasion by activating PI3K/AKT pathway $(31,32,44)$. It is unclear whether the PI3K/AKT pathway is involved in the FN-enhanced melanoma cell survival. Here, we demonstrated that extracellular FN activated PI3K/AKT signaling in a time-dependent manner and inhibiting PI3K/AKT signaling almost abrogated the pro-survival effect of $\mathrm{FN}$, indicating that activation of this pathway contributed to the FN-mediated melanoma cell survival.

In summary, we found that Dex upregulated the expression of FN protein in melanoma cells through a GR-mediated increase in protein stability. In melanoma cells, upregulation of FN contributed to the adhesion-promoting effect of Dex, thereby promoting cell survival and enhancing cell resistance to chemotherapy through activation of PI3K/AKT pathway. These new findings increase our understanding of the mechanism responsible for GC promotion of melanoma cell adhesion and survival.

\section{Acknowledgements}

This study was supported by the National Natural Science Foundation of China (no. 81472690) and the Scientific Research and Technology Development Program of Guilin (no. 2016012702-3).

\section{References}

1. Andersen BL, Yang HC, Farrar WB, Golden-Kreutz DM, Emery CF, Thornton LM, Young DC and Carson WE III: Psychologic intervention improves survival for breast cancer patients: A randomized clinical trial. Cancer 113: 3450-3458, 2008.

2. Chida Y, Hamer M, Wardle J and Steptoe A: Do stress-related psychosocial factors contribute to cancer incidence and survival? Nat Clin Pract Oncol 5: 466-475, 2008.

3. Kim-Fuchs C, Le CP, Pimentel MA, Shackleford D, Ferrari D, Angst E, Hollande F and Sloan E: Chronic stress accelerates pancreatic cancer growth and invasion: A critical role for beta-adrenergic signaling in the pancreatic microenvironment. Brain Behav Immun 40: 40-47, 2014.

4. Sloan EK, Priceman SJ, Cox BF, Yu S, Pimentel MA, Tangkanangnukul V, Arevalo JM, Morizono K, Karanikolas BD, $\mathrm{Wu} \mathrm{L}$, et al: The sympathetic nervous system induces a metastatic switch in primary breast cancer. Cancer Res 70: 7042-7052, 2010

5. Powell ND, Tarr AJ and Sheridan JF: Psychosocial stress and inflammation in cancer. Brain Behav Immun 30 (Suppl): S41-S47, 2013.

6. Beck IM, Vanden Berghe W, Vermeulen L, Yamamoto KR, Haegeman G and De Bosscher K: Crosstalk in inflammation: The interplay of glucocorticoid receptor-based mechanisms and kinases and phosphatases. Endocr Rev 30: 830-882, 2009.

7. Lin KT and Wang LH: New dimension of glucocorticoids in cancer treatment. Steroids 111: 84-88, 2016.

8. Rutz HP: Effects of corticosteroid use on treatment of solid tumours. Lancet 360: 1969-1970, 2002.
9. Kriegler AB, Bernardo D and Verschoor SM: Protection of murine bone marrow by dexamethasone during cytotoxic chemotherapy. Blood 83: 65-71, 1994.

10. Herr I, Ucur E, Herzer K, Okouoyo S, Ridder R, Krammer PH, von Knebel Doeberitz M and Debatin KM: Glucocorticoid cotreatment induces apoptosis resistance toward cancer therapy in carcinomas. Cancer Res 63: 3112-3120, 2003.

11. Sui M, Chen F, Chen Z and Fan W: Glucocorticoids interfere with therapeutic efficacy of paclitaxel against human breast and ovarian xenograft tumors. Int J Cancer 119: 712-717, 2006.

12. Zhang C, Beckermann B, Kallifatidis G, Liu Z, Rittgen W, Edler L, Büchler P, Debatin KM, Büchler MW, Friess H and Herr I: Corticosteroids induce chemotherapy resistance in the majority of tumour cells from bone, brain, breast, cervix, melanoma and neuroblastoma. Int J Oncol 29: 1295-1301, 2006.

13. Zhang C, Marmé A, Wenger T, Gutwein P, Edler L, Rittgen W, Debatin KM, Altevogt P, Mattern J and Herr I: Glucocorticoid-mediated inhibition of chemotherapy in ovarian carcinomas. Int J Oncol 28: 551-558, 2006.

14. Schoneveld OJ, Gaemers IC and Lamers WH: Mechanisms of glucocorticoid signalling. Biochim Biophys Acta 1680: 114-128, 2004.

15. Herr I, Gassler N, Friess H and Büchler MW: Regulation of differential pro- and anti-apoptotic signaling by glucocorticoids. Apoptosis 12: 271-291, 2007.

16. Warny M, Keates AC, Keates S, Castagliuolo I, Zacks JK, Aboudola S, Qamar A, Pothoulakis C, LaMont JT and Kelly CP: p38 MAP kinase activation by Clostridium difficile toxin A mediates monocyte necrosis, IL-8 production and enteritis. J Clin Invest 105: 1147-1156, 2000.

17. Paul A, Wilson S, Belham CM, Robinson CJ, Scott PH, Gould GW and Plevin R: Stress-activated protein kinases: Activation, regulation and function. Cell Signal 9: 403-410, 1997.

18. Zaidel-Bar R and Geiger B: The switchable integrin adhesome. J Cell Sci 123: 1385-1388, 2010.

19. Ponta H, Sherman L and Herrlich PA: CD44: From adhesion molecules to signalling regulators. Nat Rev Mol Cell Biol 4: 33-45, 2003.

20. Hynes RO: Integrins: Bidirectional, allosteric signaling machines. Cell 110: 673-687, 2002.

21. Winograd-Katz SE, Fässler R, Geiger B and Legate KR: The integrin adhesome: From genes and proteins to human disease. Nat Rev Mol Cell Biol 15: 273-288, 2014.

22. Zaidel-Bar R, Itzkovitz S, Ma'ayan A, Iyengar R and Geiger B: Functional atlas of the integrin adhesome. Nat Cell Biol 9: 858-867, 2007.

23. Ahadome SD, Zhang C, Tannous E, Shen J and Zheng JJ: Small-molecule inhibition of Wnt signaling abrogates dexamethasone-induced phenotype of primary human trabecular meshwork cells. Exp Cell Res 357: 116-123, 2017.

24. Guller S, Wozniak R, Leibman MI and Lockwood CJ: Negative regulation of placental fibronectin expression by glucocorticoids and cyclic adenosine 3',5'-monophosphate. Ann N Y Acad Sci 734: 132-142, 1994.

25. DobosJ,Kenessey I,TímárJ andLadányi A: Glucocorticoid receptor expression and antiproliferative effect of dexamethasone on human melanoma cells. Pathol Oncol Res 17: 729-734, 2011.

26. Banciu M, Metselaar JM, Schiffelers RM and Storm G: Liposomal glucocorticoids as tumor-targeted anti-angiogenic nanomedicine in B16 melanoma-bearing mice. J Steroid Biochem Mol Biol 111: 101-110, 2008.

27. Lai S, Piras F, Spiga S, Perra MT, Minerba L, Piga M, Mura E, Murtas D, Demurtas P, Corrias M, et al: Nestin and vimentin colocalization affects the subcellular location of glucocorticoid receptor in cutaneous melanoma. Histopathology 62: 487-498, 2013.

28. Nakagawa Y, Nakayama H, Nagata M, Yoshida R, Kawahara K, Hirosue A, Tanaka T, Yuno A, Matsuoka Y, Kojima T, et al: Overexpression of fibronectin confers cell adhesion-mediated drug resistance (CAM-DR) against 5-FU in oral squamous cell carcinoma cells. Int J Oncol 44: 1376-1384, 2014.

29. Schmidt $S$ and Friedl P: Interstitial cell migration: Integrin-dependent and alternative adhesion mechanisms. Cell Tissue Res 339: 83-92, 2010.

30. Leiss M, Beckmann K, Girós A, Costell M and Fässler R: The role of integrin binding sites in fibronectin matrix assembly in vivo. Curr Opin Cell Biol 20: 502-507, 2008.

31. Li XP, Zhang XW, Zheng LZ and Guo WJ: Expression of CD44 in pancreatic cancer and its significance. Int J Clin Exp Pathol 8: 6724-6731, 2015. 
32. McFarlane S, McFarlane C, Montgomery N, Hill A and Waugh DJ: CD44-mediated activation of $\alpha 5 \beta 1$-integrin, cortactin and paxillin signaling underpins adhesion of basal-like breast cancer cells to endothelium and fibronectin-enriched matrices. Oncotarget 6: 36762-36773, 2015.

33. Clark AS, West K, Streicher S and Dennis PA: Constitutive and inducible Akt activity promotes resistance to chemotherapy, trastuzumab, or tamoxifen in breast cancer cells. Mol Cancer Ther 1: 707-717, 2002.

34. Larue L and Bellacosa A: Epithelial-mesenchymal transition in development and cancer: Role of phosphatidylinositol 3'kinase/AKT pathways. Oncogene 24: 7443-7454, 2005.

35. Polivka J Jr and Janku F: Molecular targets for cancer therapy in the PI3K/AKT/mTOR pathway. Pharmacol Ther 142: 164-175, 2014.

36. Valles SL, Benlloch M, Rodriguez ML, Mena S, Pellicer JA, Asensi M, Obrador E and Estrela JM: Stress hormones promote growth of B16-F10 melanoma metastases: An interleukin 6- and glutathione-dependent mechanism. J Transl Med 11: 72, 2013.

37. Collinson FJ, Lam TK, Bruijn WM, de Wilt JH, Lamont M, Thompson JF and Kefford RF: Long-term survival and occasional regression of distant melanoma metastases after adrenal metastasectomy. Ann Surg Oncol 15: 1741-1749, 2008.
38. Flaherty DC, Deutsch GB, Kirchoff DD, Lee J, Huynh KT, Lee DY, Foshag LJ, Bilchik AJ and Faries MB: Adrenalectomy for metastatic melanoma: Current role in the age of nonsurgical treatments. Am Surg 81: 1005-1009, 2015.

39. McKeown-Longo PJ and Etzler CA: Induction of fibronectin matrix assembly in human fibrosarcoma cells by dexamethasone. J Cell Biol 104: 601-610, 1987.

40. Nimmer D, Bergtrom G, Hirano H and Amrani DL: Regulation of plasma fibronectin biosynthesis by glucocorticoids in chick hepatocyte cultures. J Biol Chem 262: 10369-10375, 1987.

41. Oliver N, Newby RF, Furcht LT and Bourgeois S: Regulation of fibronectin biosynthesis by glucocorticoids in human fibrosarcoma cells and normal fibroblasts. Cell 33: 287-296, 1983.

42. Zollinger AJ and Smith ML: Fibronectin, the extracellular glue. Matrix Biol 60-61: 27-37, 2017.

43. Benbrook DM and Masamha CP: The pro-survival function of Akt kinase can be overridden or altered to contribute to induction of apoptosis. Curr Cancer Drug Targets 11: 586-599, 2011.

44. Onodera Y, Teramura T, Takehara T and Fukuda K: Hyaluronic acid regulates a key redox control factor Nrf2 via phosphorylation of Akt in bovine articular chondrocytes. FEBS Open Bio 5: 476-484, 2015. 